\title{
Nomadisme : Présentation générale
}

Hélène Claudot-Hawad

\section{OpenEdition}

Journals

Édition électronique

URL : https://journals.openedition.org/encyclopedieberbere/2749

DOI : $10.4000 /$ encyclopedieberbere. 2749

ISSN : 2262-7197

\section{Éditeur}

Peeters Publishers

\section{Édition imprimée}

Date de publication : 5 octobre 2012

Pagination : 5572-5577

ISBN : 978-90-429-2718-6

ISSN : $1015-7344$

\section{Référence électronique}

Hélène Claudot-Hawad, « Nomadisme : Présentation générale », Encyclopédie berbère [En ligne], 34 2012, document N63, mis en ligne le 15 décembre 2020, consulté le 17 février 2022. URL : http:// journals.openedition.org/encyclopedieberbere/2749; DOI : https://doi.org/10.4000/ encyclopedieberbere. 2749

Ce document a été généré automatiquement le 17 février 2022.

(c) Tous droits réservés 


\title{
Nomadisme : Présentation générale
}

\author{
Hélène Claudot-Hawad
}

1 Les "Berbères" dans l'imagerie coloniale ont été associés à la sédentarité - en référence au modèle kabyle - et opposés à la figure du nomade incarnée par les « Arabes ». Or, dans les régions arides et semi-arides du nord de l'Afrique, la majorité du monde amazighophone est nomade ou l'était encore récemment.

2 Avant la colonisation, en effet, toutes les zones de steppe et de désert étaient régies par les nomades. Au Sahara central et sur ses franges sahéliennes, ce sont les Imuhagh, Imajaghen ou Imushagh (les «Touaregs » des étrangers), qui ont façonné le milieu, que ce soit politiquement, économiquement, géographiquement, socialement et culturellement. L'essentiel de la toponymie* saharienne est berbérophone. Ces vastes zones administrées par les grands pôles politiques touaregs jusqu'au début du XXe siècle ont été divisées dans les années 1960 entre cinq Etats nés des indépendances africaines, les uns se déclarant "arabo-musulmans" - Algérie, Libye -, les autres « sahéliens »- Mali, Niger, actuel Burkina-Faso. Plus au nord, en Algérie, dans les zones steppiques, le semi-nomadisme, pratiqué entre autres par les grandes confédérations Chaoui des Aurès*, « concernait les $9 / 10^{e}$ de la population et dominait directement ou non toutes les activités économiques » jusqu'à la fin du XIXe siècle (Boukhobza 1989). A l'ouest, du côté marocain, les ensembles confédéraux berbères Sanhaja* de l'Atlas, du nord au sud, comme les Aït Idrassen, les Zemmour*, Aït Atta*... ont dominé l'arrièrepays qui ne reconnaissait pas l'autorité du Makhzen. Ils ont étendu leur emprise territoriale parfois jusqu'aux cités représentant l'autorité du Sultan, comme par exemple les Zemmour au début du $\mathrm{XX}^{\mathrm{e}}$ siècle qui arrivaient aux portes de Meknès, de Rabat, et jusqu'aux murs de Salé (Lesne 1966).

3 Le nomadisme n'a aucun rapport avec l'errance et correspond à des déplacements réguliers associés à une territorialité nomade particulière. Celle-ci correspond à une organisation sociale de type confédéral et souvent acéphale qui a inspiré à certains auteurs (Gellner en premier lieu) des analyses reprenant en l'adaptant la théorie de la « segmentarité » développée par Evans-Pritchard (1937) au sujet des Nuer. En fait, la notion de société segmentaire a été élaborée en premier lieu par Durkheim (1897) à partir des données fournies par Hanoteau et Letourneux dans La Kabylie et les coutumes 
kabyles ( 1 ère édition: 1873). Ces entrecroisements de données compatibles avec un même modèle montrent que, au-delà des différences de mode de vie, les Berbères sédentaires et nomades partagent certains principes fondamentaux dans la manière de concevoir et de gérer l'ordre social, s'exprimant dans des organisations politiques qui varient selon les contextes historiques et locaux, mais qui présentent de profondes affinités structurelles.

Divers types de nomadisme existent ou co-existent dans ces régions désertiques: grands nomades chameliers, éleveurs de petit bétail dont les mouvements pendulaires sont plus restreints, agro-pasteurs qui pratiquent plutôt la transhumance saisonnière. L'aptitude à la mobilité s'accompagne d'un matériel adapté et de techniques éprouvées. Elle correspond également à l'acquisition de savoirs spécifiques indispensables à la navigation dans les étendues arides (notamment des connaissances géographiques, hydrauliques, botaniques, zoologiques, climatiques, techniques...) dont l'apprentissage commence dès le plus jeune âge. L'amplitude des déplacements équivaut souvent à celle de la position sociale des groupements, les grands nomades se trouvant en général au sommet de la hiérarchie. Dans tous les cas de figures, le nomadisme chez les Berbères et plus largement chez les nomades du nord de l'Afrique - est lié au pastoralisme*. L'élevage - des moutons*, des chèvres*, des vaches, des chevaux* et des chameaux*, dont la répartition varie selon les ressources régionales -, se combine à d'autres activités économiques : l'agriculture saisonnière, l'artisanat parfois très réputé (telles la peausserie ou la métallurgie touarègues qui circulaient dans tout le Sahara), enfin l'économie des transports et du commerce. La cueillette et la chasse forment des activités d'appoint. La puissance guerrière et politique de certains groupes leur assurait par ailleurs le versement d'un tribut en échange de leur protection. Au Sahara où la démographie est un enjeu important, des populations d'origines diverses ont été agrégées aux grands pôles politiques nomades et berbérisées sur le plan linguistique et culturel, certains groupes conservant parallèlement leur langue d'origine. C'est le cas par exemple des arabophones et des pularophones du Gourma (région relevant de l'actuel Mali), appartenant à l'ancienne confédération touarègue de la Tademmekat (dirigée par les Iwellemeden* de l'ouest). La double inscription culturelle et linguistique de ces groupes apparait dans le nom qu'ils se donnent eux-mêmes, littéralement "Arabes des Touaregs» ou "Peuls des Touaregs ». Dans ces territoires, l'hégémonie nomade a été un vecteur puissant de diffusion de la culture et de la langue berbères.

5 Les nomades berbérophones par ailleurs ont toujours évolué dans un paysage linguistique plus diversifié que celui des sédentaires. S'il arrive que les femmes soient monolingues, par contre, les hommes parmi les grands nomades parlent souvent trois à cinq langues (arabe, haoussa, peul, songhay...) leur permettant d'exercer leurs activités caravanières ou marchandes dans les régions qu'ils fréquentent. 


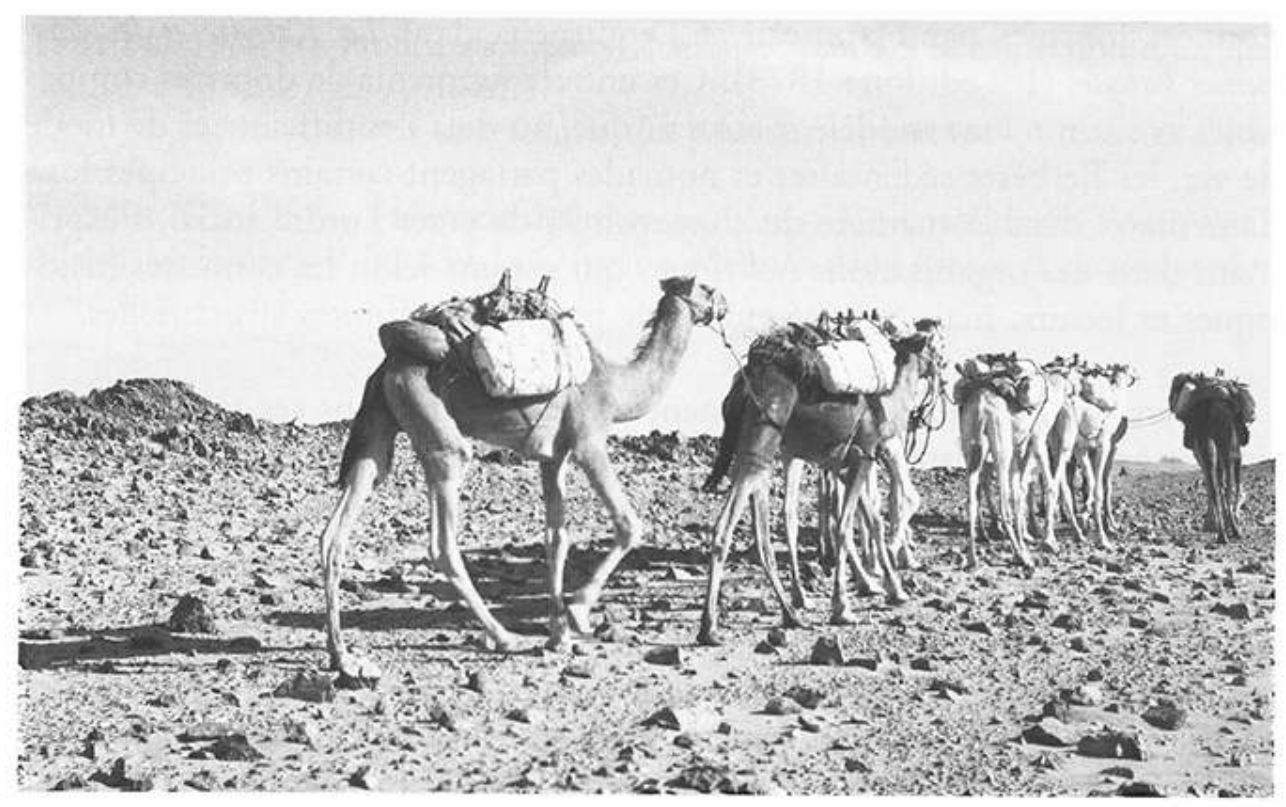

Photo 1. Caravane de Sel, nOvembre 1964 Près de tamanrasset

Cliché M. Gast.

6 Une complémentarité étroite existait autrefois entre nomades et sédentaires, chacun exploitant des niches écologiques distinctes. Le passage de l'un à l'autre mode de vie s'observe par ailleurs dans les deux sens au cours de l'histoire. La période contemporaine a imposé ou, dans le meilleur des cas, favorisé la sédentarisation comme seule voie d'accès à la " modernité », tandis que les législations étatiques - coloniales puis postcoloniales - ont abouti concrètement à la dépossession territoriale des nomades et à leur cantonnement dans des espaces limités qui ne leur permettent plus d'exercer la gestion rationnelle et la protection des ressources fragiles qu'ils appliquaient aux milieux désertiques dans lesquels ils vivent. Peu de nomades ont résisté au vaste mouvement de sédentarisation entamé à la fin du XIX ${ }^{e}$ siècle sous la pression des Etats et des nouvelles économies orientées vers le profit des villes et de la métropole (exploitation de l'alpha, expansion des cultures céréalières, monoculture d'exportation, extractions minières entrainant également la pollution des nappes phréatiques...). Ces nouveaux enjeux économiques ont progressivement chassé les nomades de leur territoire ou les ont ghettoïsés, transformant leurs déplacements en transhumance et leurs campements en villages. De nombreux auteurs ont abordé ces questions, soulignant tous la réduction drastique si ce n'est la disparition des nomades dans diverses régions, leur prolétarisation et leur paupérisation extrême (voir entre autres: Boukobza 1982 et 1989, Casciarri 2006, Chellig 2005, Claudot-Hawad 2006, Keenan 2006, Lefébure 1986, Rachik 2000, Zaïnabi 1989).

7 De l'antiquité à la période contemporaine, les pouvoirs citadins se sont heurté aux nomades en cherchant à les contenir et ont livré d'eux des portraits effrayants et sauvages qui traduisent surtout la crainte que ces derniers pouvaient leur inspirer. Les nomades y sont rejetés aux marges de l'humanité, opposés terme à terme au portrait du sédentaire qui pour ces urbains incarne seul la civilisation et le progrès.

Le contrôle des routes et l'accès au territoire et à ses ressources - végétales, animales, hydrauliques et, dans l'économie mondiale actuelle, minérales - est à la source de la plupart des conflits entre groupes nomades eux-mêmes, entre pasteurs et agriculteurs, 
entre pouvoirs anciens fondés sur la mobilité nomade et pouvoirs nouveaux de type sédentaire et urbain, entre ce qui est devenu aujourd'hui le « local » et ce qui incarne le "global », inversant l'ordre précolonial des espaces désertiques.

9 La sédentarisation a souvent précipité les changements d'identités linguistique, sociale, culturelle. Cependant, le maintien de la vie et des activités nomades ne correspond pas nécessairement à la pérennisation de la langue ou de la culture berbères. Ainsi, les Maures* (Bidan), qui à la période médiévale appartiennent au même ensemble culturel que les Touaregs, sont devenus « Arabes » et arabophones suivant un lent processus de mutation identitaire qui s'étend $d u \mathrm{XV}^{\mathrm{e}}$ au XVIII ${ }^{\mathrm{e}}$ siècle. Cependant, ils partagent encore beaucoup de traits culturels avec les Touaregs, par exemple au niveau de leur culture matérielle, conditionnée non seulement par le mode de vie nomade, mais aussi par des conceptions esthétiques et symboliques clairement ancrées dans la logique géométrique berbère.

Enfin, on relève trois grands types d'habitats chez les Berbères nomades, en fonction des matériaux de construction utilisés : les tentes en laine dominent au nord, tandis que les tentes en peau et les tentes en nattes prévalent au sud chez les Touaregs. Il existe enfin des huttes de paille, constructions plus éphémères qui sont laissées sur place, ainsi que des aménagements d'abris naturels (grottes, arbres, greniers et enclos en pierre), que l'on retrouve chaque année quand le cycle nomade ramène à l'étape saisonnière du parcours. Le choix des matériaux dépend de l'environnement et des ressources disponibles (la laine de mouton renforcée souvent avec des poils de chèvres étant abondante au nord tandis qu'au sud, le pelage des animaux est ras). Les constructions se différencient également par rapport à la forme des charpentes, les unes organisées autour d'un piquet central, les autres privilégiant les arceaux. La tente est investie d'une dimension symbolique importante, apparaissant comme un microcosme qui reflète l'ordre de l'univers. Beaucoup d'éléments structurants de la tente nomade se retrouvent d'ailleurs dans la maison* sédentaire dont certaines appellations sont remarquablement stables d'une région à l'autre (voir par exemple Laoust 1920 : chap. I et II). Le champ sémantique des termes concernant l'habitation en berbère permet de nombreux rapprochements entre régions pourtant extrêmement éloignées et prouve à la fois la stabilité de certaines racines lexicales berbères et la variabilité des significations qui leur sont prêtées en fonction des contextes. 


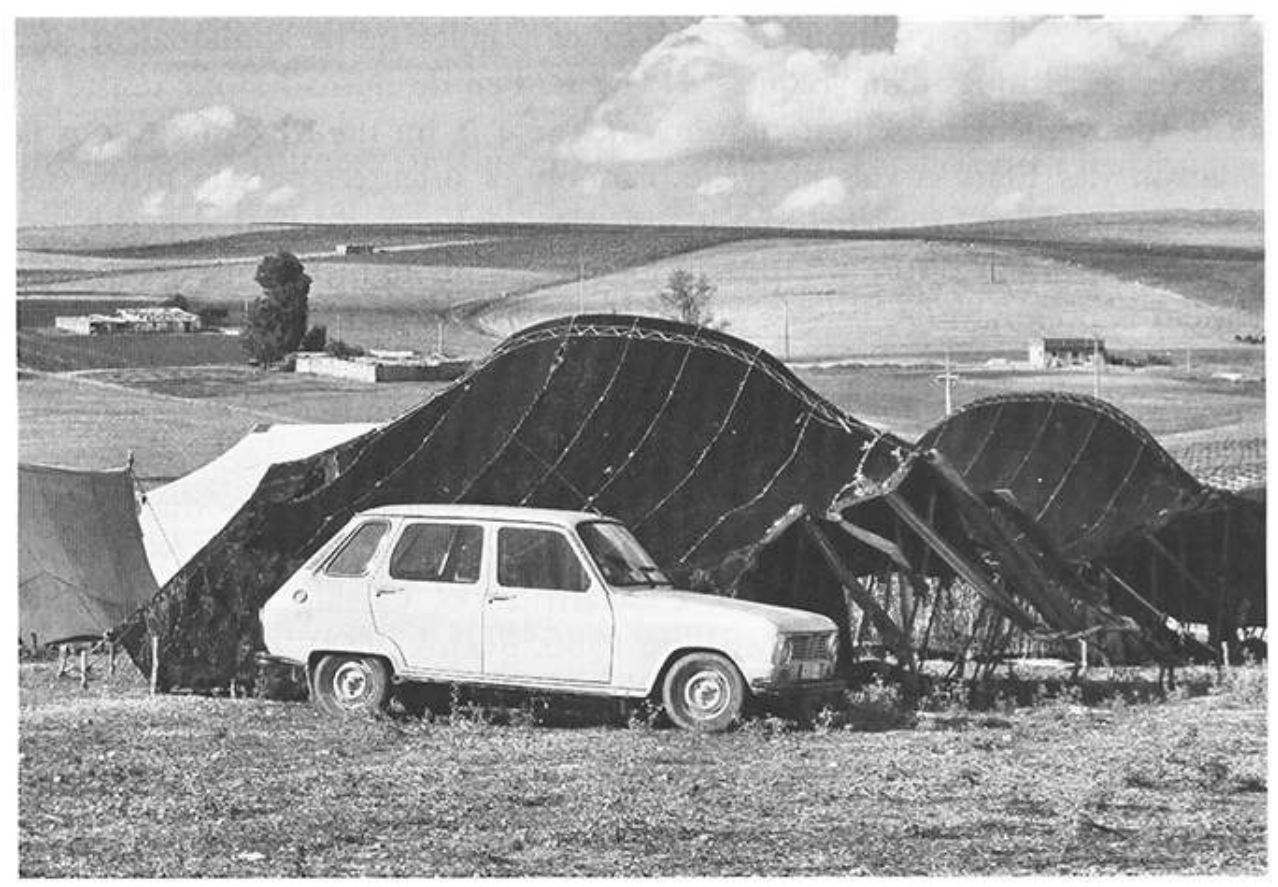

Photo 2. TENTE (AXAM) DES IMAZIgHEN, OULMES (MOYEN-ATLAS).

Cliché Tahir Aït Awari

\section{BIBLIOGRAPHIE}

AG FONI Eghleze, 1979 - L'impact socio-économique de la sécheresse dans le cercle de Kidal, Bremen, Borda.

AG MAHMOUD M., 1992 ( $2^{\mathrm{e}}$ éd.) - Le Haut Gourma central, Montpellier, CEFE.

BELLIL R., 2006, « Nomades 'arabes' et sédentaires 'berbères' au Sahara dans une étude de C. Sabatier (1891)», in Claudot-Hawad H. (dir.), p. 57-74.

BERNARD A. \& LACROIX N., 1906 - Évolution du nomadisme en Algérie, Alger, Jourdan, 342 p.

BOETSCH G. et FERRIÉ J.-N., 1989 - « Le paradigme berbère : approche de la logique classificatoire des anthropologues français du XIX siècle », Bulletins et mémoires de la Société d'anthropologie de Paris, I/3-4, p. 257-267.

вочкновZА М., 1982 - L'agro-pastoralisme traditionnel en Algérie. De l'ordre tribal au désordre colonial, Alger, OPU, $458 \mathrm{p}$.

BOUKновZA M., 1989 - « Société nomade et Etat en Algérie », Politique africaine, nº 34, p. 7-18.

CASCIARRI B., 2006 - « Ait Unzar pastoralists of south-eastern Maroco », in Chatty D. (dir.), p. 393-426. 
CHASSEY F. (de), 1996 - « Anthropologie des sociétés traditionnelles mauritaniennes : les Maures, pasteurs-nomades arabo-berbères, et les Toucouleurs ou Halpularen, agriculteurs sédentaires négro-africains ", Cahiers d'URBAMA, $\mathrm{n}^{\circ} 12$, p. 81-89.

CHATTY D. (dir.), 2006 - Nomadic Societies in the Middle East and North Africa. Entering the 21st Century, Leiden, Brill, $1060 \mathrm{p}$.

CHELLIG N., 2005 - Du nomadisme, Essai d'anthropologie historique sur les relations entre les pouvoirs dans la société algérienne, Alger, CNRPAH, 269 p. et annexes.

CLAUDOT-HAWAD H. (dir.), 2006a - Berbères ou Arabes? Le tango des spécialistes, Paris, Non-Lieu, 300 p. CLAUDOT-HAWAD H., 2006b - « A Nomadic Fight against Immobility : the Tuareg in the Modern States », in Chatty D. (dir.), 2006, p. 654-681.

DURKHEIM E., 1967 [1897] - De la division du travail social, Paris, PUF.

EVANS-PRITCHARD E. E., 1994 [1937] - Les Nuer. Description des modes de vie et des institutions politiques d'un peuple nilote, Paris, Gallimard, 1994.

GALlOY P., 1957 - « Nomadisation et sédentarisation dans les cercles de Goundam et de Tombouctou, Mission d'étude et d'aménagement du Niger », Etudes de Géographie Humaine, Niamey.

GELLNER E., 1969 - Saints of the Atlas, Londres, Weidenfeld \& Nicolson, 316 p.

hanoteau A. et LetourneuX A., 1893 [1873], La Kabylie et ses coutumes, Paris, Challamel.

HART D., 1984 - The Ait Atta of Southern Marocco. Daily Life and Recent History, Cambridge Middle East and North African Studies Press.

KEENAN J., 2006 - « Sedentarization and changing patterns of social organization amongst the Tuareg of Algeria », in CHATTY D. (dir.), p. 917-939.

LA CHAPELle F. (Lt de) - « Une cité de l'oued Dra' sous le protectorat des nomades », Hespéris, 1929, $\mathrm{n}^{\circ}$ 9, p. 29-42.

LAOUST E., 1920 - Mots et choses berbères, Paris, Challamel, $531 \mathrm{p}$.

LEFÉBURE Cl., 1986 - « Ait Kebbache, impasse sud-est. L'involution d'une tribu marocaine exclue du Sahara », ROMM, $\mathrm{n}^{\circ}$ 41-42, p. 69-76.

LEHURAUX, Cpt. L. 1948 - Où va le nomadisme en Algérie ? Alger, Baconnier, 184 p.

LESNE M., 1966 - Les Zemmour. Essai d'histoire tribale (édité en trois parties), ROMM, 1966, nº 2, p. 111-154;1967, nº 3, p. 97-132;1967, nº 4, p. 31-80.

RACHIK H., 2000 - Comment rester nomade, Beyrouth, Afrique-Orient.

ZAÏNABI A., 1989 - « Vers une disparition rapide du nomadisme au Sahara marocain : le cas du Dra' Moyen », in Le nomade, l'oasis et la ville, Cahiers d'URBAMA, XX, Univ. de Tours, p. 49-68.

\section{INDEX}

Mots-clés : Commerce, Economie, Sahara 